Objectives: To understand the mechanism and frequency of the positive temporal artery of GCA in ${ }^{18}$ F-FDG-PET.

Methods: This was a retrospective chart review of 3 patients with giant cell arteritis who underwent all of ${ }^{18} \mathrm{~F}$-FDG-PET, MRI, CT angiogram, ultrasound and histopathologic evaluation of temporal arteries. To investigate the cases of FDG-PET positive temporal arteries in GCA, a systematic literature review was done in Pubmed using ${ }^{18}$ F-FDG-PET AND ("temporal arteritis" OR "giant cell arteritis").

Results: A case report: An 85-year-old male presented with muscle weakness. Three years before admission, he experienced pain in his neck and both extremities. Rheumatoid arthritis was diagnosed at another hospital, and the patient was treated with methotrexate and prednisolone, which resolved his symptoms. The medication was discontinued after 2 years. Two months before admission he began coughing due to interstitial pneumonitis, and later developed muscle weakness and difficulty walking. On admission a physical examination revealed swelling of the bilateral temporal arteries. A blood test showed accelerated ESR at $54 \mathrm{~mm} / \mathrm{h}$ and a CRP of $4.6 \mathrm{mg} / \mathrm{dL}$. Ultrasound showed an increased diameter, hypoechoic wall thickening (halo), stenosis, and calcification of the arterial wall of the temporal artery. A contrast CT showed wall thickening in the affected segments, calcification, stenosis and enhancement of the soft tissue around the temporal artery. An ${ }^{18} \mathrm{~F}$-FDG-PET scan indicated increased uptake of ${ }^{18} \mathrm{~F}$-FDG at the temporal arteries whereas no uptake was found in the other arteries including the aorta and carotid arteries. A biopsy of the temporal artery revealed necrotizing vasculitis with a few giant cells and the formation of a microscopic neutrophilic abscess. When compared with 2 other cases of temporal arteritis with a negative ${ }^{18} \mathrm{~F}$-FDG uptake, the severity of inflammation and the number of giant cells in the present case were not remarkably high. A systematic literature review identified 4 cases of ${ }^{18} \mathrm{~F}$-FDG-PET positive temporal arteries in 454 cases of GCA of 82 papers. Of note, large arteries were positive for ${ }^{18} \mathrm{~F}$-FDG-PET in 253 cases of GCA in the same series.

Conclusions: The hierarchy for FDG uptake by resting inflammatory cells is neutrophil $>$ macrophage $=$ lymphocyte in vitro, indicating that the sites where neutrophils dominate are more likely to be visualized by ${ }^{18} \mathrm{~F}$-FDG PET in vivo. In addition to the known usefulness of ${ }^{18} \mathrm{~F}$-FDG-PET in visualizing inflammation in large vessels, the increased uptake of ${ }^{18} \mathrm{~F}$-FDG to the temporal artery may help to identify the neutrophilic inflammation.

Disclosure of Interest: None declared

DOI: 10.1136/annrheumdis-2017-eular.4171

\section{AB0567 THE LONG TERM HEALTH RELATED QUALITY OF LIFE (HRQOL) IMPACT ON GIANT CELL ARTHRITIS (GCA) PATIENT}

\section{H. Horiuchi. General Medicine, Kurashiki Central Hospital, Okayama, Japan}

Background: Health related quality of life (HRQOL) of Giant cell Arthritis (GCA) patients is improtant because it adds depth to our understanding of how a disease and its treatments affect them. Though only one previous study has addressed the short term impact of GCA on HRQOL, the long term impact has not fully been investigated.

Objectives: The aim of our study is to assess the long term HRQOL outcomes of GCA patients using the Japanese version of EuroQol 5 Dimention (EQ-5D). Methods: 40 GCA patients who admitted to our hospital from November 2004 to June 2014 were enrolled. All patients were received prednisolone over 2 years. Patients who had lost their eyesight were excluded because vision concern for HRQOL nor it is difficult to mesure by using EQ-5D. This is a retrospective study and deta were collected by telephone interview. Patients evluated their health status using five dimensions. The EQ-5D score were calculated based on the Japanese version of the value set. Primary outcome is the mortality rate and the norm of EQ-5D score. As a secondary analysis, we classified the patients as follows and compare the mortality rate and EQ-5D score between two groups.

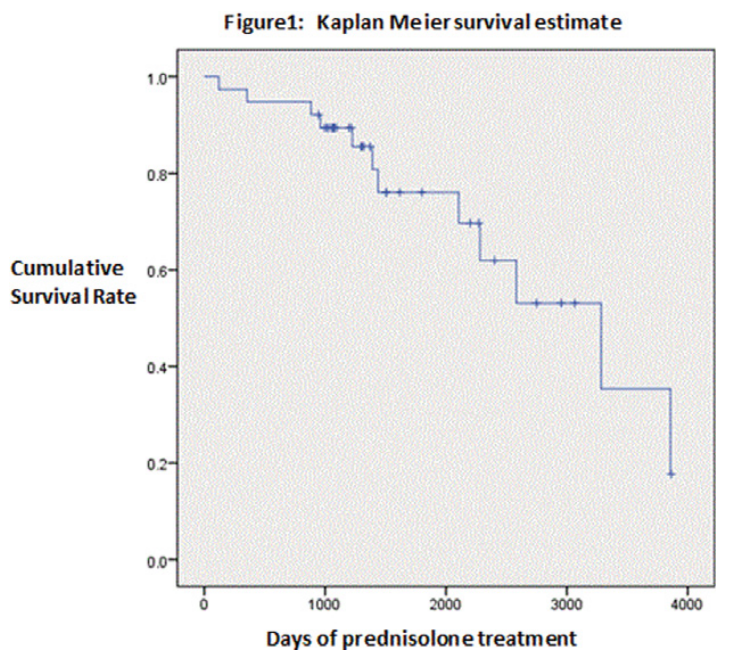

Group 1: GCA patients who received prednisolone over 5 years.

Group 2: GCA patients who received prdnisolone less than 5 years.

Results: There were 16 male (40\%) and 24 female (60\%). The median age was $83.5(95 \% \mathrm{Cl} 79.24-87.76)$. The mortality rate was $37.5 \%$ (15 patients). Kaplan-Meier curve is shown in Figure1. The median EQ-5D score was 0.746 (95\% confidence interval [Cl] 0.852-0.640) and it is lower than Japanese norm ( 0.853 in male and 0.808 in female). In the secondary analysis, the mortality rate was $56.0 \%$ (14 patients) in Group1 and 6.6\% (1 patient) in Group2. It was significantly higher in Group $1(P<0.05)$. The median EQ-5D score was 0.764 $(95 \% \mathrm{Cl} 0.579-0.870)$ in Group1 versus 0.768 (95\% Cl 0.621-0.915) in Group2. There was no statistically significant difference between them $(P=0.813)$.

Conclusions: This study focused on GCA patients with prednisolone therapy over 2 years. It showed that EQ-5D was decreased compared with the Japanese norm but it didn't clearly decrease after 2 years of treatment. These findings suggest the value of measuring health status in GCA patients by EQ-5D at least first 2 years of treatment, because it would allow comprehensive evaluation of the patient's health condition and add another dimension to the subjective symptoms and laboratory data.

References:

[1] Arthritis and Rheumatism Vol.49,No6, December 15,2003,pp819-825.

[2] J Neuroophthalmol. $2001 \mathrm{Dec} ; 21(4): 266-73$.

Disclosure of Interest: None declared

DOI: 10.1136/annrheumdis-2017-eular.3691

\section{AB0568 APPLICATION OF OZONATED WATER IN ORAL ULCER PATIENTS WITH BEHCET'S DISEASE}

H. Miao, L. Zhang, G. Zhang, K. Xu. Rheumatology, Shanxi Dayi Hospital, Taiyuan, China

Background: Behcet's disease is a systemic vasculitis disease of unknown etiology. Oral ulcer is the most common symptoms, the ulcer is various, the swelling and pain is obvious, and it is easy to recurrent, these symptoms affected the patient's diet and life seriously.Therefore, it is important to care the patients with Behcet's disease of oral ulcer.The ozonated water had many functions such as immune activation and immune regulation, it can induce the production of many cytokines,promote the repairment of oral epithelium. At the same time,the ozonated water had a direct effect on the nerve endings,it played a better analgesic effect, it was widely used in oral mucositis which induced by chemotherapy.But there were few studies about application ozonated water in the patients with Behcet's disease of oral ulcer, it was not widely used in clinical practice.

Objectives: Apply the ozonated water in patients with Behcet's disease,observe the effectiveness in patients with Behcet's disease of oral ulcer.

Methods: From June 2014 to June 2016,82 cases of hospitalized patients with Behcet's disease were randomly divided into study group and control group $(n=41)$, the control group used nursing method of gentamycin sulfate solution and nystatin solution gargle alternatively,the research group using ozonated water gargle,observe and evaluate the healing effect of two groups of patients.

Results: The research group had significant improvement in healing oral ulcer and relieving pain $(\mathrm{P}<0.05)$.

Conclusions: Ozonated water had a perfect effect in patients with Behcet's disease of oral ulcer.

References:

[1] Wainstein J, Feldbrin Z, Boaz, M et al. Defficacy of ozone-oxygen therapy for the treatment of diabetic foot ulcers[J].Diabetes Technol Ther 2011;31(12):12551260.

[2] Kim KI, Kim JW, Lee HJ, et al. Recombinant human epidermal growth factor on oral mucositis induced by intensive chemotherapy with stem cell transplantation [J]. Am J Hematol 2013;88(2):107-112.

[3] Xiaotong Yi.The effect of ozonated water on the anti-inflammatory and wound healing of infected wound $[\mathrm{J}]$. Asia Pacific Traditional Chinese medicine, 2012;8(4):331-350.

[4] Xia Wu. Effect of different nursing methods in patients with Behcet's disease of oral ulcer [J]. Southwest Military 2010;12(3):571-572.

[5] Yuan Li, Zeju Yu, Shuling Yang, et al. Effect of ozone water treatment in oral ulcer which induced by radiotherapy[J]. Chinese Journal of nursing, 2012:18(24):2956-2957.

Disclosure of Interest: None declared

DOI: 10.1136/annrheumdis-2017-eular.3371

\section{AB0569 CHANGE IN SERUM LEVEL OF SOLUBLE E-SELECTIN AND MMP-9 IN CHILDREN WITH KAWASAKI DISEASE}

H. Zeng. Department of Pediatric Allergy, Immunology and Rheumatolog, Guangzhou Women and Children's Medical Center, Guangzhou, China

Objectives: Kawasaki disease (KD)is a kind of febrile disorder without definite etiology. Coronary artery aneurysms are the major complication of Kawasaki disease (KD). r11le pathogenesis of the vascular damage remains unknown. This study Wfl8 conducted to explore the pathophysiological role of E-selectin (ES), CMatrix metalloproteinase 9 (MMP-9) in KD.and to look for the evidenee of direct relationship between the plasma levels of ES,MMP-9 and the incidence of the coronary artery lesion (CAL). 